\title{
Coactivation of Motoneurons Regulated by a Network Combining Electrical and Chemical Synapses
}

\author{
Lorena Rela and Lidia Szczupak \\ Laboratorio de Fisiología y Biología Molecular, Departamento de Fisiología, Biología Molecular y Celular, Facultad de Ciencias Exactas y Naturales, \\ Universidad de Buenos Aires, Ciudad Universitaria, 1428 Buenos Aires, Argentina
}

Electrical transmission among neurons has been considered a mechanism to synchronize neuronal activity, and rectification provides a mechanism to confine the flow of signals among the connected neurons. The question is how this type of transmission operates within complex neuronal networks. In the leech, the neurons located in position 151 of the midbody ganglion map are connected to virtually every motoneuron via rectifying electrical synapses that pass negative current to the motoneurons. These are nonspiking neurons, and here we have labeled them NS neurons. The goal of this investigation has been to assess their role in regulating motor activity and how rectifying electrical synapses contribute to the function of motor networks.

The coupling between NS neurons and motoneurons was voltage sensitive: it increased as motoneurons were depolarized. In addition, excitation of motoneurons evoked hyperpolarizing synaptic responses in NS neurons, the amplitude of which depended on the membrane potential of the latter and on the motoneuron firing frequency. This hyperpolarization was mediated by chemical transmission through an interneuronal layer that spanned the nerve cord. These interactions established a feedback loop between NS and motoneurons that was regulated by the membrane potential of NS. This mechanism was responsible for the uncoupling between otherwise electrically coupled motoneurons. In this way, the NS neurons can act as "electrical neuromodulators," modifying the interaction of other neurons, depending on the activity of the system as a whole.

Key words: gap junctions; electrical rectification; rectifying electrical synapses; motor control; leech; nonspiking

\section{Introduction}

The role that electrical synaptic transmission plays in neuronal networks is not well understood (Bennett, 1997; Dermietzel, 1998; Kiehn and Tresch, 2002). Its investigation requires a routine performance of paired intracellular recordings. Probably because this procedure is more amenable in invertebrate nervous systems, the contribution of electrical transmission to the properties of particular neural circuits has been well characterized in various invertebrates (Furshpan and Potter, 1959; Nicholls and Purves, 1970; Muller and Scott, 1981; Graubard and Hartline, 1987; Edwards et al., 1998), whereas in the vertebrate nervous system electrical coupling has been considered of importance mainly at early postnatal stages, at which it is widely observed (Peinado et al., 1993). In recent studies, however, electrically coupled neurons have been found in different regions of the CNS (Ishimaru and Williams, 1996; Galarreta and Hestrin, 1999; Gibson et al., 1999; Koos and Tepper, 1999; Galarreta and Hestrin, 2001a; Schmitz et al., 2001), and it has been proposed that electrical coupling contributes to spike synchronization (Gibson et al., 1999; Mann-Metzer and Yarom, 1999; Tamás et al., 2000; Alvarez et al., 2002), to coordination

Received Aug. 5, 2002; revised Sept. 25, 2002; accepted Sept. 27, 2002.

This study was supported by grants from Fogarty International Research and Collaboration Award (National Institutes of Health), Human Frontier Science Program, and Agencia de Promoción Científica y Tecnológica. We thank Dr. D. Edwards, Dr. G. González Burgos, Dr. G. Murer, Dr. L. Pozzo-Miller, and Dr. A. Schinder for helpful discussion of this manuscript. We also thank M. Rodríguez for assistance with statistical analysis and D. Toledo for graphics assistance.

Correspondence should be addressed to Lorena Rela, CC. 4992, 1000 Capital Federal, 1428 Buenos Aires, Argentina.E-mail: Irela@bg.fcen.uba.ar.

Copyright $\odot 2003$ Society for Neuroscience $\quad 0270-6474 / 03 / 230682-11 \$ 15.00 / 0$ of postsynaptic inhibitory potentials (Beierlein et al., 2000), and to coincidence detection (Galarreta and Hestrin, 2001b). In particular, electrical coupling has been observed as a common feature of networks of inhibitory neurons (Galarreta and Hestrin, 2001a; Landisman et al., 2002).

Electrical transmission plays a recognized role in the leech nervous system, where this type of connectivity has been characterized among well identified neurons. Electrical coupling among motoneurons (Stuart, 1970), sensory neurons (Acklin, 1988), interneurons (Muller and Scott, 1981), and neuromodulatory neurons (Hagiwara and Morita, 1962; De Miguel et al., 2001) and in sensorimotor pathways (Nicholls and Purves, 1970) can be readily recognized. One particular case is constituted by the pair of neurons identified in position 151 in the midbody ganglion map (Wadepuhl, 1987). These neurons have been identified as nonspiking neurons, which we have labeled NS neurons, and they are connected to virtually every excitatory motoneuron in the ganglion through rectifying electrical connections (Wadepuhl, 1989) that transmit only negative current to the motoneurons and allow a graded regulation of the firing frequency of the latter (Iscla et al., 1999). The scheme shown in Figure 1 describes the network formed by a pair of NS neurons and the motoneurons that derived from previous knowledge. It suggests that the NS neurons are located in a key position to influence the whole motor system in a global and graded manner.

Here we have investigated how the NS neurons act on the motor system. The results indicate that the NS neurons are key elements in a feedback mechanism that regulates the coupling among motoneurons. This feedback mechanism was formed by a web of electrical (rectifying and nonrectifying) and chemical syn- 


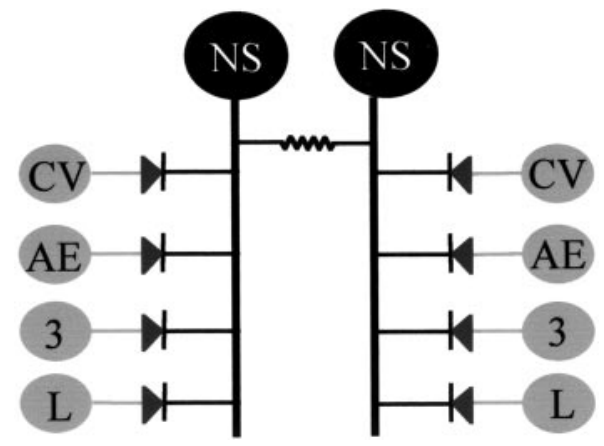

Figure 1. Scheme of the divergent connections between the NS neurons and the motoneurons. The scheme represents a circuit showing the divergent path from the bilateral pair of NS neurons (black) to the excitatory motoneurons $\mathrm{CV}, \mathrm{AE}$, cell 3 , and $\mathrm{L}$ ( gray) within a single ganglion. The NS cells are coupled to each other through nonrectifying electrical synapses and to the motoneurons through rectifying electrical synapses. $C V$, Circular ventral excitor; $A E$, annulus erector; 3 , cell 3 dorsal longitudinal excitor; $L$, longitudinal excitor.

apses that included an interneuronal layer that spanned through the leech nervous system. The knowledge derived from this particular system uncovers a neuronal network in which a neuron can act as an "electrical neuromodulator," modifying the interaction of other neurons depending on the activity of the system as a whole.

\section{Materials and Methods}

Biological preparation. Hirudo medicinalis, weighing 2-5 gm, were obtained from a commercial supplier (Leeches USA, Westbury, NY) and
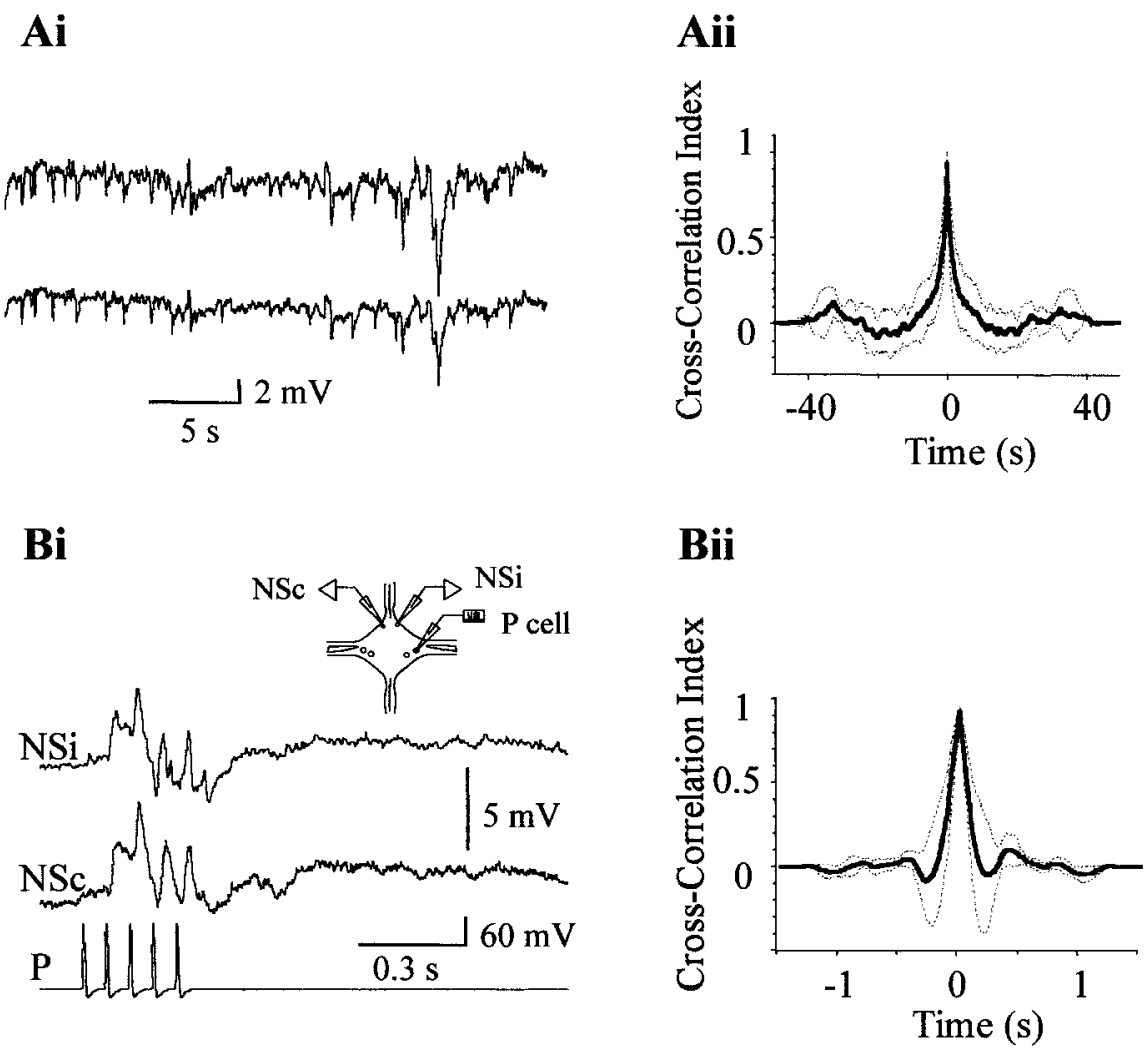

Figure 2. Simultaneous recordings from pairs of NS neurons within a ganglion. Ai, Representative simultaneous recordings of the spontaneous activity of both NS neurons in a single ganglion. Aii, Average cross-correlogram. The cross-correlograms (bin size $0.5 \mathrm{msec}$ ) of six different pairs of traces were averaged. The dotted lines represent the $95 \%$ confidence interval for the mean cross-correlation index. Bi, Representative simultaneous recordings of the synaptic responses of both NS neurons, evoked by a train of action potentials $(15 \mathrm{~Hz})$ elicited in a P cell in a single ganglion. The scheme on top represents a ganglion and the recording configuration. NSi and NSc denote NS cells ipsilateral and contralateral to the stimulated P cell, respectively. Bii, Average of three cross-correlograms (bin size $0.1 \mathrm{msec}$ ). The dotted lines represent the $95 \%$ confidence interval for the mean cross-correlation index. maintained at $15^{\circ} \mathrm{C}$ in artificial pond water. The animals were not fed for at least 1 month before dissection. Individual ganglia, or chains of ganglia, where stated, were dissected out of the animal and pinned to Sylgard (Dow Corning) in a superfusion chamber at room temperature $\left(\sim 20^{\circ} \mathrm{C}\right)$. The sheath covering the ganglion was dissected away, leaving the neurocell bodies exposed to the external solution.

Solutions. The ganglia were bathed in standard saline solution of the following composition (in mM): $115 \mathrm{NaCl}, 4 \mathrm{KCl}, 1.8 \mathrm{CaCl}_{2}, 1 \mathrm{MgCl}_{2}, 5.4$ Tris base, 10 glucose, $\mathrm{pH}$ 7.4. To block synaptic transmission (Nicholls and Baylor, 1968) we used a solution with a high $\mathrm{Mg}^{2+} / \mathrm{Ca}^{2+}$ ratio $(20$ $\mathrm{mm} \mathrm{MgCl}_{2}$ and $1 \mathrm{mM} \mathrm{CaCl}_{2}$ ). To discriminate between monosynaptic and polysynaptic connections (Nicholls and Purves, 1970) between neurons, we used a solution with a high concentration of divalent cations (10 $\mathrm{mm} \mathrm{MgCl}_{2}$ and $10 \mathrm{~mm} \mathrm{CaCl}_{2}$ ). In these solutions, the osmolarity was kept constant by reducing the $\mathrm{NaCl}$ concentration. The different solutions were applied through the perfusion system.

Electrophysiological recordings. Neuronal activity was recorded using intracellular glass microelectrodes connected to an Axoclamp 2B amplifier (Axon Instruments, Foster City, CA) operating in the current-clamp configuration. Microelectrodes were pulled from borosilicate capillary tubing (FHC, Brunswick, ME) and filled with a $3 \mathrm{~m}$ potassium acetate solution. Electrodes with a resistance of $40-60 \mathrm{M} \Omega$ were selected. The recordings were digitized using a Digidata 1320 interface and acquired using Clampex protocols (pClamp 8.0.2, Axon Instruments) at sampling frequencies of $5-10 \mathrm{kHz}$. The neurons were identified by their location, size, electrophysiological properties, and synaptic connections (Muller et al., 1981; Granzow et al., 1985). Where stated, the membrane potential of the neurons was shifted to different values by injecting DC current through the bridge-balanced recording electrode. The recording electrode was also used, where indicated, to apply square current pulses into the neurons. Pressuresensitive $(\mathrm{P})$ cells were stimulated by trains (5-25 $\mathrm{Hz}, 0.5-2 \mathrm{sec}$ ) of suprathreshold step pulses (2-4 $\mathrm{nA}, 5 \mathrm{msec}$ ) delivered by a stimulator (Master-8; AMPI, Jerusalem, Israel) and triggered by the acquisition software. To measure the input resistance of NS and motoneurons, we injected square current steps $(-0.2 \mathrm{nA}, 1 \mathrm{sec})$ through the recording electrode. Its value was calculated by dividing the amplitude of the voltage deflection at steady state by the amplitude of the current step.

Data analysis. The recordings were analyzed using commercial software (Axograph 4.5, Axon Instruments). The synaptic responses were quantified by measuring the maximum amplitude from baseline or by measuring the time integral, as indicated in the corresponding figure legends. The coupling coefficient between two cells was calculated as $d / D$, where $D$ is the deflection produced by a square pulse of negative current injected into one of the cells (presynaptic) and $d$ is the deflection produced in the coupled cell (postsynaptic). Cross-correlation analysis was performed using Axograph (the bin size is indicated in the corresponding figure legends). Curve fitting was achieved using commercial software (Kaleidagraph 3.0.2, Abelbeck Software).

The results are expressed as the average value \pm their SEM, and the number of neurons, or pairs of neurons, studied is expressed between brackets $(n)$. Statistical significance of the differences was determined by $t$ tests and paired two-factor ANOVA.

\section{Results}

\section{Electrical connectivity between}

NS neurons

The pairs of NS neurons, present in each ganglion, are interconnected via electrical junctions (Wadepuhl, 1989). To establish 
A

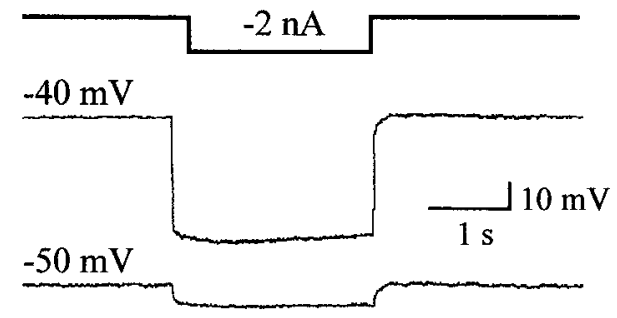

B

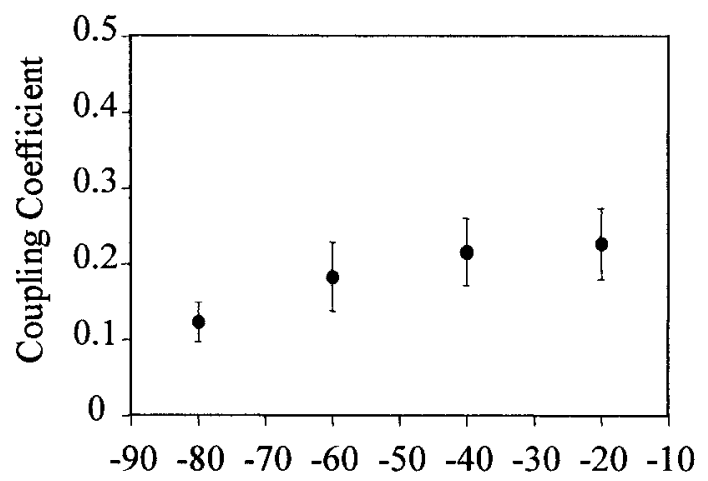

Membrane Potential of Presynaptic Cell (mV)

Figure 3. Coupling between pairs of NS neurons as a function of the membrane potential. $A$, Representative recordings showing the responses of a pair of NS neurons to a current step injected into one of them (represented by the square step line on top). We define the injected NS neuron as presynaptic (top trace) and the second NS neuron as postsynaptic (bottom trace). The membrane potential at which the cells were set is indicated on the left of each trace. $B$, The graph displays the coupling coefficient between pairs of NS neurons as a function of the membrane potential of the presynaptic neuron. The presynaptic cell was shifted to different membrane potentials, whereas the postsynaptic NS neuron was set at $-50 \mathrm{mV}$. The symbols and error bars indicate mean and SEM, respectively $(n=7)$. Statistical analysis used two-factor ANOVA with repeated measures.

the extent of coupling between the bilateral pair, we analyzed paired recordings of both NS cells while displaying spontaneous and evoked electrophysiological activities.

NS neurons generally displayed spontaneous changes in membrane potential that were similarly reflected in the two contralateral somata. Figure $2 \mathrm{Ai}$ shows an example of paired recordings, in which both NS neurons displayed a series of hyperpolarizing shifts in their membrane potential of highly similar appearance. These spontaneous changes in membrane potential probably originated by synaptic input onto the NS neurons. Cross-correlation analysis of these recordings and of another five different pairs of NS neurons, displaying various spontaneous activities, showed a high correlation index. The average crosscorrelogram is shown in Figure $2 \mathrm{Aii}$, and the average delay of the individual cross-correlograms was of $0.2 \pm 0.2 \mathrm{msec}$. The similarity between the recordings of the two bilateral homologs indicates that the synaptic sites were electrically equidistant from both somata. To confirm this interpretation, we analyzed the responses to evoked synaptic activity. Stimulation of a mechanosensory cell sensitive to pressure ( $\mathrm{P}$ cell) evokes synaptic responses in the NS neurons that are composed of both depolarizing and hyperpolarizing phases (Marin-Burgin and Szczupak, 2000). Simultaneous recordings of both NS homologs revealed that the evoked synaptic potentials detected in both somata were

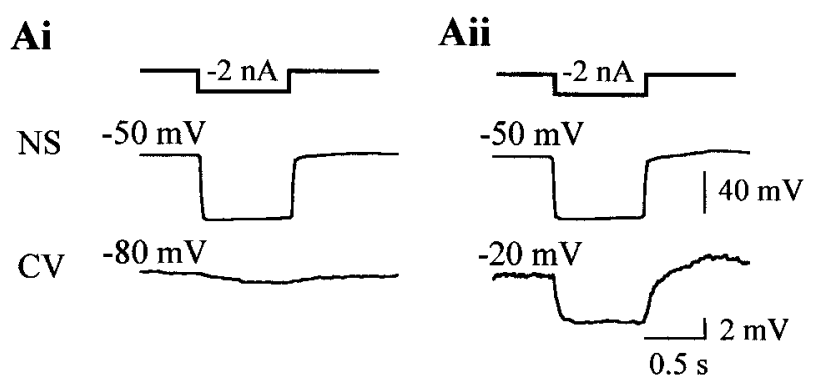

B

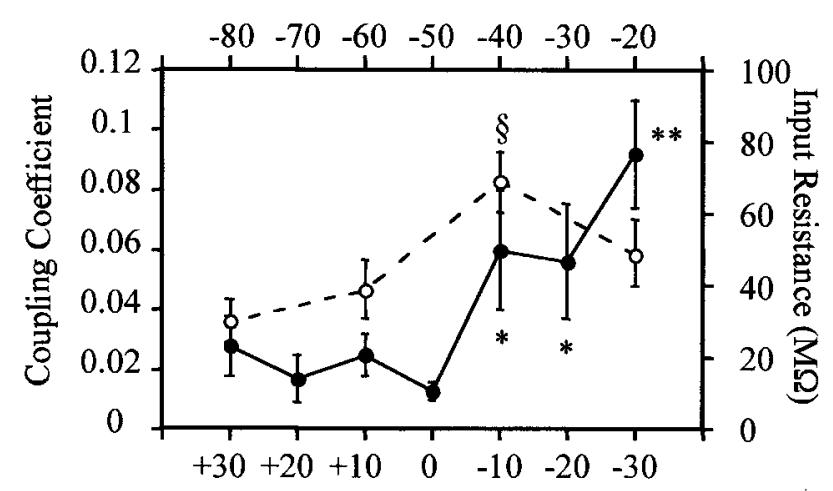

Difference in potential between somata $(\mathrm{mV})$

C

NS Membrane Potential $(\mathrm{mV})$

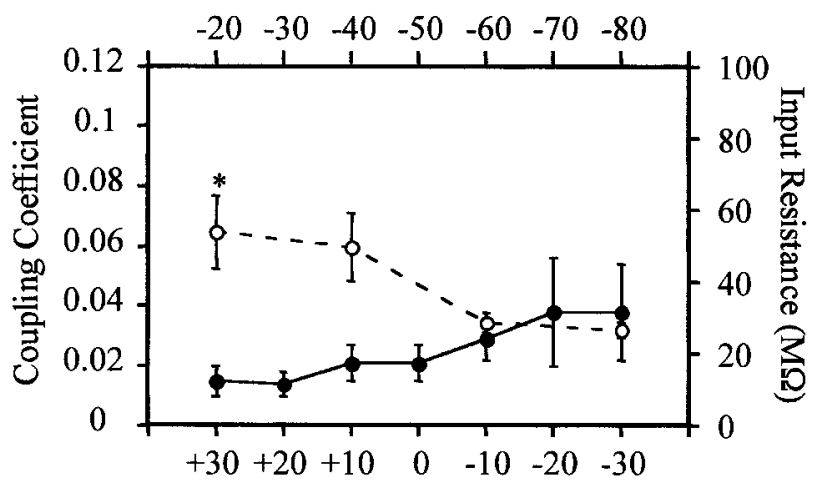

Difference in potential between somata $(\mathrm{mV})$

Figure 4. Coupling between NS neuron and CV motoneuron as a function of the transjunctional potential. $A$, Representative recordings showing the responses of an NS neuron and a CV motoneuron to a hyperpolarizing square pulse (represented by the square step line on top) injected in the NS neuron. The membrane potential of the NS neuron was set at $-50 \mathrm{mV}$, whereas the membrane potential of the CV motoneuron was set at $-80 \mathrm{mV}$ (Ai) or $-20 \mathrm{mV}$ (Aii). B, The graph shows the coupling coefficient between $\mathrm{CV}$ and NS neurons $(-)$ and the input resistance of the $\mathrm{CV}$ motoneurons $(\mathrm{O})$ as a function of the membrane potential of the $\mathrm{CV}$ motoneuron (top axis) and of the difference in membrane potential between the NS neurons and the $\mathrm{CV}$ motoneurons (bottom axis), measured at the somata $\left(V \mathrm{~m}_{\mathrm{NS}}-V \mathrm{~m}_{\mathrm{CV}}\right) \cdot{ }^{\S} p<0.01$ (compared with each one of the other data points of the same curve); ${ }^{*} p<0.01$ and ${ }^{* *} p<0.001$ (compared with the value obtained at no potential difference between somata).C, The graph shows the coupling coefficient between the CV motoneurons and the NS neurons $(O)$ and the input resistance of the NS neurons $(O)$ as a function of the membrane potential of the NS neuron (top axis) and of the difference in membrane potential between the CV motoneurons and the NS neurons (bottom axis), measured at the somata $\left(V \mathrm{~m}_{\mathrm{NS}}-V \mathrm{~m}_{\mathrm{CV}}\right) .{ }^{*} p<0.01$ (compared with the value obtained at a membrane potential of $-80 \mathrm{mV}$ ). The coupling coefficient was calculated as the amplitude of the response displayed by the motoneuron (postsynaptic) over that displayed by the NS neuron (presynaptic). The symbols and error bars indicate mean and SEM, respectively ( $n=15$, for each case). Statistical analysis used two-factor ANOVA with repeated measures. 
$\mathbf{A}$

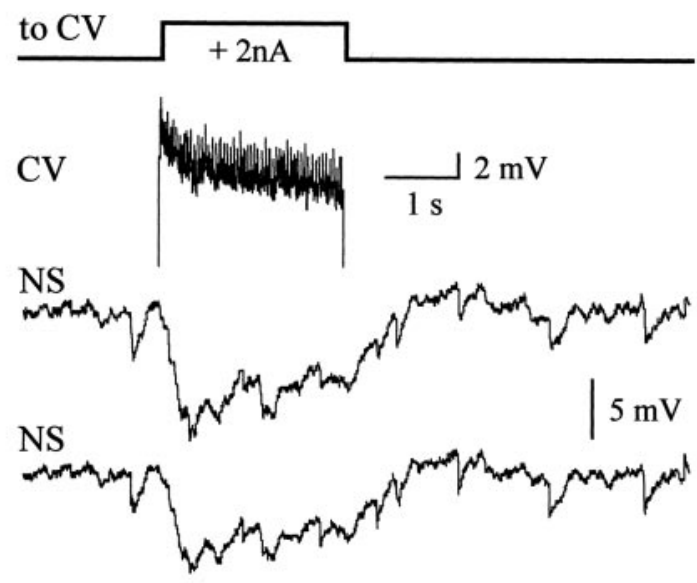

B

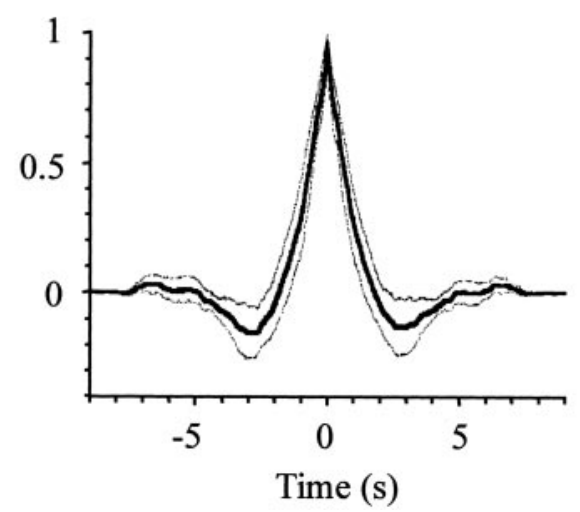

Figure 5. Responses of NS neurons to motoneuron excitation. $A$, The two bottom traces show representative recordings of the simultaneous responses of both NS neurons within a single ganglion to the stimulation of a CV motoneuron with a square current pulse (square step line on top). The top trace shows the recording of the $\mathrm{CV}$ motoneuron during the pulse injection. The small action potentials recorded in the soma reflect the passive propagation of fully developed action potentials initiated at an electrically distant site (Stuart, 1970). B, Average crosscorrelogram. The cross-correlograms (bin size $0.2 \mathrm{msec}$ ) of 11 different pairs of traces were averaged. The broken lines represent the $95 \%$ confidence interval for the mean cross-correlation index.

also very similar (Fig. $2 \mathrm{Bi}$ ). In this experimental configuration, we observed a consistent temporal shift between the two recordings. Cross-correlation analysis (Fig. 2 Bii) showed that the responses of the NS neurons contralateral to the stimulated P cells were delayed with respect to the ipsilateral ones by $3 \pm 1 \mathrm{msec}$ $(n=6)$.

To test whether this tight coupling between homologous NS neurons was sensitive to the voltage across the junctions (junction potential), we measured the coupling coefficient (see Materials and Methods) as the presynaptic cell was set at different membrane potentials, whereas the postsynaptic cell was set at around $-50 \mathrm{mV}$. The results described in Figure 3 indicate that the coupling coefficient was not significantly affected by shifting the neuronal potential to different values.

These results strongly suggest that the pair of NS neurons operate as a unit for a wide variety of signals because of the efficiency of the electrical coupling between the contralateral homologs through nonrectifying junctions.
A
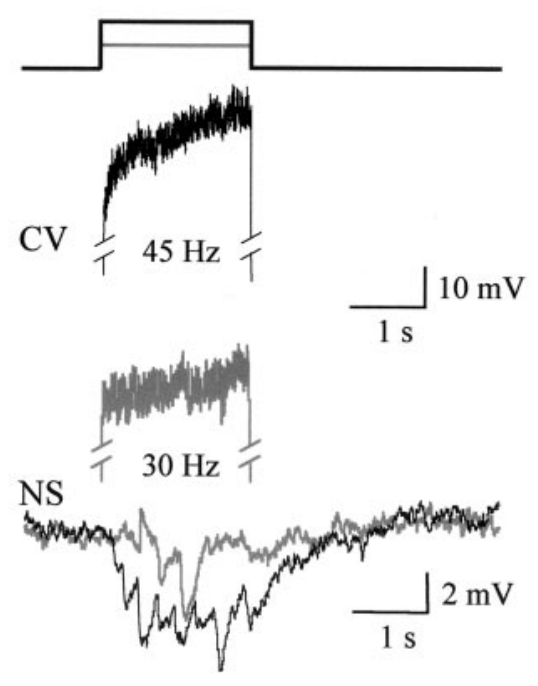

B

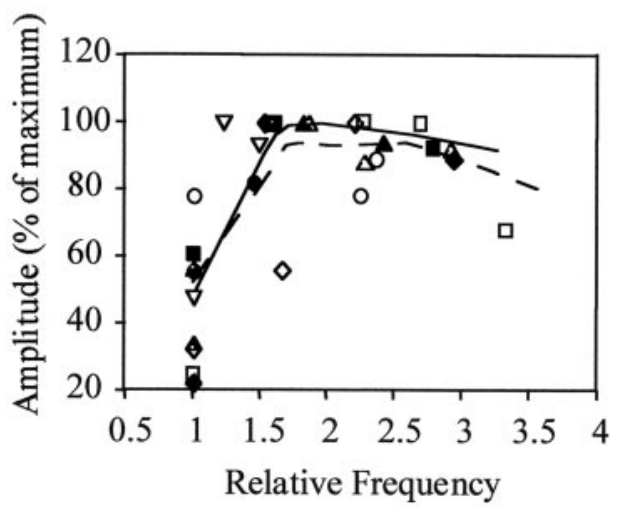

Figure 6. Responses of NS neurons as a function of the spike frequency in the motoneuron. $A$, Representative recordings of an NS neuron to the activation of a CV motoneuron. The two top traces correspond to a CV motoneuron, which was induced to fire at two different frequencies (indicated under the traces) by the application of square current pulses (indicated by the square step lines on top). The superimposed traces on the bottom correspond to the respective responses of the NS neuron. Black and gray traces indicate the corresponding recording pairings. B, Amplitude of the NS response as a function of the spike frequency of the motoneurons, stimulated with current steps of $0.5,1,1.5$, and $2 \mathrm{nA}$. Firing frequencies were normalized to the one observed in response to the smallest current step $(0.5 \mathrm{nA})$. Open symbols represent five $\mathrm{CV}$ motoneurons; filled symbols represent four AE motoneurons. The curves are weighted fittings using the locally weighted least squared error method performed with Kaleidagraph. Broken line indicates CV data; solid line indicates AE data.

Electrical connectivity between NS neurons and motoneurons Previous reports suggest that NS neurons are connected to virtually every excitatory motoneuron in the ganglion (Wadepuhl, 1989 ) and also to the serotonergic Retzius neurons (MarinBurgin and Szczupak, 1998). These electrical synapses allow the conduction of negative current from NS neurons to motoneurons and positive current from motoneurons to NS neurons. This feature suggests that the electrical junctions are sensitive to the voltage across the junctions. To test this hypothesis we applied a procedure similar to that used in analyzing the connectivity between the NS homologs.

Because the NS neurons are on the ventral side of the ganglion, we studied their interaction with the two motoneurons that are located on the same side (Stuart, 1970): the AE motoneurons (responsible for the erection of the skin annuli) and the CV mo- 
toneurons (responsible for the contraction of the circular muscles). The coupling between NS and AE, or NS and CV, was analyzed in terms of the transmission of negative current from the NS neurons to the motoneurons. The neurons were shifted to different membrane potentials, and the junction potential was estimated for each case. Because of spatial attenuation, the difference between the potentials of the two somata is an overestimation of the junction potential, and it was considered only as indicative of the polarity and the magnitude of the junction potential at the actual junction sites.

Variations in the membrane potential of the motoneuron produced significant $(p<0.001)$ changes in the coupling coefficient (Fig. 4A). The coupling coefficient was maintained at a low constant value in the range from -80 to $-50 \mathrm{mV}$ and strongly increased when the motoneuron was shifted to more depolarized values (Fig. $4 B, 0$ ). Manipulation of the membrane potential could have affected the input resistance of the motoneuron, and this, in turn, could have modified its space constant, causing an apparent change in the coupling coefficient. To evaluate this possibility we measured the input resistance of the CV neurons as a function of their membrane potential (Fig. $4 \mathrm{~B}, \mathrm{O}$ ). The input resistance of the motoneuron had a maximum value around -40 $\mathrm{mV}$ and decreased significantly $(p<0.01)$ at more depolarized or hyperpolarized potentials. Thus, the increase in the coupling coefficient observed between -80 and $-40 \mathrm{mV}$ could have been influenced by the concomitant increase in input resistance observed at this membrane potential range. However, the coupling increased with further depolarization of the motoneuron, although its input resistance decreased. These data suggest that, in addition to the influence of the input resistance on the coupling coefficient, the junction conductance was influenced by the junction potential, increasing as the NS neuron side became more negative. Variations in the membrane potential of the NS neurons did not produce statistically significant changes in the coupling coefficient (Fig. 4C, 9 ), although it showed a tendency to increase as the NS potential was shifted to potentials more negative than the resting value (approximately $-50 \mathrm{mV}$ ). The input resistance of the NS cells decreased as they were hyperpolarized (Fig. $4 C, \bigcirc)(p<0.01)$, and thus it is possible that estimation of the coupling at hyperpolarized potentials was conditioned by the negative influence of a decrement in the input resistance.

\section{Motoneurons inhibit NS neurons through a \\ polysynaptic pathway}

The current flow from motoneurons to NS neurons could only be clearly seen in a high $\mathrm{Mg}^{2+}$ solution, which impairs chemical transmission, because in normal saline the preponderant response of the NS neurons to excitation of the motoneurons is a series of inhibitory synaptic potentials. When the AE or the CV motoneurons were stimulated with a positive current step, both homologous NS neurons showed a highly similar series of synaptic hyperpolarizing potentials (Fig. $5 A$ ). The cross-correlograms of paired NS recordings (Fig. $5 B$ ) evidenced a delay between the responses of $3.7 \pm 1.6 \mathrm{msec}$, indicating that the NS contralateral to the excited motoneuron presented the shortest latency. One should remember that leech motoneurons send their branches toward the periphery through the contralateral roots, and they extend most of their neuritic arborizations in the contralateral hemiganglion. The amplitude of the hyperpolarizing responses increased with the firing frequency of the motoneuron, but we did not observe a one-to-one correlation of the individual postsynaptic potentials with the motoneuron spikes (Fig. 6).

The amplitude of the response was also dependent on the
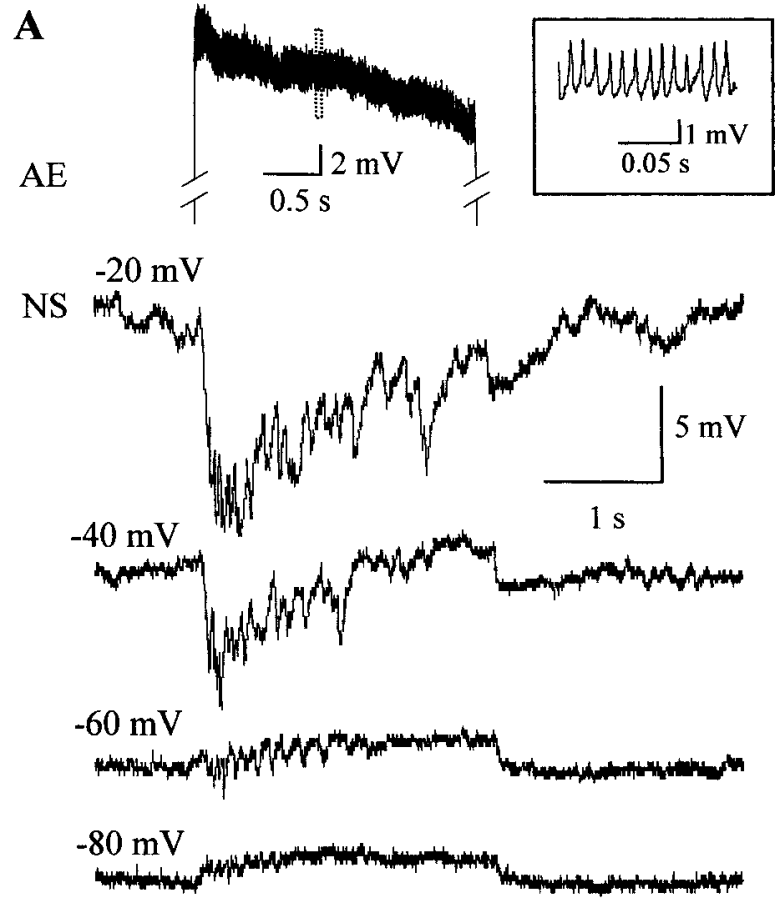

B

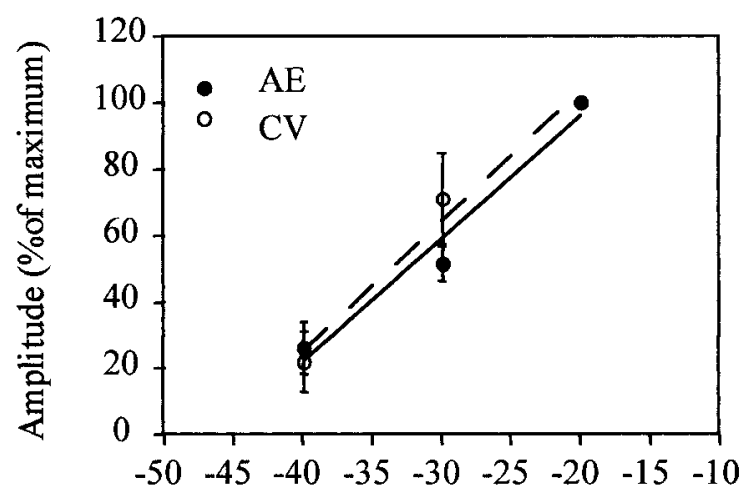

Membrane Potential of NS neuron (mV)

Figure 7. Responses of NS neurons to the activation of motoneurons at different membrane potentials of NS. A, Representative recordings of an NS neuron, set at different membrane potentials (indicated on the left) as an AE motoneuron (top trace) was stimulated with a square step pulse. Only one of the motoneuron traces is shown displaying the activity during the stimulation period. The inset shows the fragment of the recording indicated by the dotted rectangle in an expanded temporal scale. Manipulation of the NS membrane potential, within the studied range, did not affect the spike frequency of the motoneuron. $B$, The graph shows the amplitude of the responses of the NS neurons to the stimulation of AE $(n=4)$ or CV $(n=4)$ motoneurons as a function of the membrane potential of the NS neuron. The stimulation protocol was like the one presented in $A$. The amplitude was measured only in those cases in which the hyperpolarization had its onset straight from baseline. The symbols and error bars indicate mean and SEM, respectively.

membrane potential of the NS neurons (Fig. 7). It increased when the neuron was depolarized, and at hyperpolarized potentials, the hyperpolarizing synaptic potentials had a very small amplitude and were superimposed on a steady depolarization (Fig. 7A), because of the transmission of positive current from the motoneuron through the electrical connections. Thus, at depolarized potentials the inhibitory chemical component of the re- 
A Normal Saline
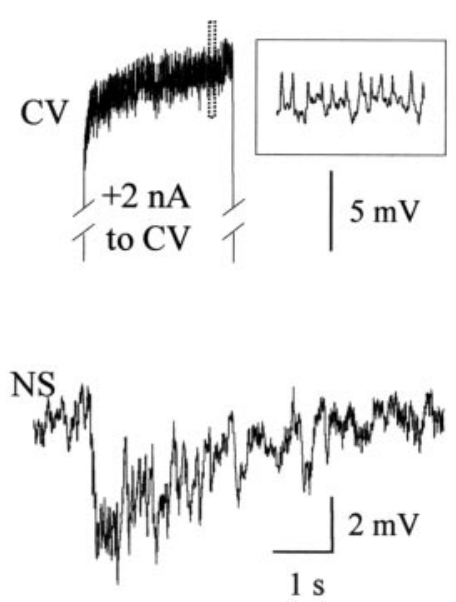

B

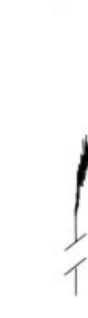

C

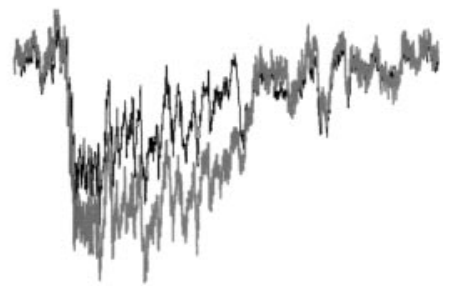

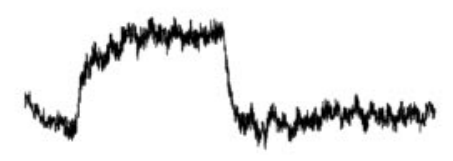

Figure 8. Responses of NS neurons to the excitation of motoneurons in a high divalents solution. $A$, Representative recordings showing the response of an NS neuron (bottom trace) to the stimulation of a CV motoneuron (top trace) during perfusion of the isolated ganglion with normal saline. The motoneuron trace displays the activity during the stimulus ( $+2 \mathrm{nA}$ square current step). The inset shows the fragment of the recording indicated by the dotted rectangle in an expanded temporal scale. $B$, The same as in $A$ after the ganglion has been perfused for 5 min with a solution containing $10 \mathrm{~mm} \mathrm{Mg}^{2+} / 10 \mathrm{~mm} \mathrm{Ca}^{2+}$. It was always possible to recover the response after a 10 min washout with normal saline ( $\left.n=5\right)$. $C$, Superposition of the trace showed in $A$ displaying the NS response in normal solution (black) and the "chemically mediated" trace ( gray). The latter was obtained after subtracting the trace in $A$ from the trace in $B$ and represents the response mediated by the chemical transmission without the component caused by the electrical transmission.

sponse counteracted the depolarizing electrical component. The projection of the linear fit between the amplitude of the synaptic response and the membrane potential (Fig. $7 B$ ) gave an estimation for the reversal potential of $-46 \pm 1 \mathrm{mV}$.

The dependence of the response amplitude on the membrane potential of the NS neuron, together with the fact that the responses are abolished in the presence of a high $\mathrm{Mg}^{2+}$ solution (Wadepuhl, 1989), indicates that the signals elicited by the motoneurons were mediated by chemical transmission through the activation of an ionic membrane conductance.

To discriminate between a monosynaptic versus a polysynaptic connection, we tested the persistence of the response in a solution with a high concentration of divalent cations (see Materials and Methods), known to impair polysynaptic pathways in the leech (Nicholls and Purves, 1970). The inhibitory responses were not observed in the solution containing high divalent cations (Fig. $8 A, B)(n=5)$, indicating that they were mediated by a spiking inhibitory interneuron(s). This condition unmasked the electrical transmission of the depolarizing signal from motoneurons to NS neurons that probably reduced the amplitude of the chemically evoked hyperpolarization (Fig. 8C). These results are consistent with the absence of a one-to-one correlation between the spikes of the stimulated motoneuron and the hyperpolarizing synaptic potentials.

Motoneurons in each segmental ganglion act locally, innervating the muscle fibers of the corresponding segment, and they do not extend branches through the nerves interconnecting the ganglia (Stuart, 1970). To test whether the inhibitory signal transmitted from motoneurons to NS neurons was confined to the ganglion of origin of these cells or could be transmitted across ganglia via the interneurons, we isolated chains of three ganglia, stimulated the $\mathrm{CV}$ or $\mathrm{AE}$ motoneurons from the anterior or posterior ganglia, and recorded from an NS neuron in the middle ganglion. AE and CV motoneurons in anterior $(n=4$, two of each type) and posterior ( $n=4$, two of each type) ganglia pro- duced inhibitory potentials in the NS neurons, which were of consistently smaller amplitude $(38 \pm 24 \%)$ than the locally generated responses, as in the examples displayed in Figure $9 \mathrm{~A}$.

This recording configuration allowed us to test the influence of the motoneurons with somata located on the dorsal side of the ganglion. The excitatory motoneurons innervating longitudinal muscle fibers, cells $\mathrm{L}$ and 3, also elicited hyperpolarizing synaptic responses in anterior $(n=3$, for each neuron) or posterior $(n=$ 3 , for each neuron) NS neurons (Fig. 9B). These responses could also be elicited by motoneurons located as far as five ganglia away from the ganglion where the soma of the NS neuron was located $(n=2$; data not shown). In contrast, stimulation of cell 1 (Fig. 9C) and cell 2 (data not shown), two inhibitory motoneurons, did not elicit any response ( $n=5$ for each type).

These results show that motoneuron activity can regulate the membrane potential of NS neurons, and this regulation can be exerted along the nerve cord.

\section{NS neurons uncouple motoneurons in a voltage-dependent way}

Because NS neurons are coupled to virtually all motoneurons (Fig. 1), it was possible that hyperpolarizing potentials elicited by excitation of one motoneuron in the NS neurons influenced, in turn, the activity of other motoneurons in the ganglion. To investigate this hypothesis we recorded from pairs of motoneurons while performing two manipulations that affected the hyperpolarizing potentials: (1) we changed the membrane potential of the NS neurons to enhance $(-20 \mathrm{mV})$ or reduce $(-80 \mathrm{mV})$ their amplitude (Fig. 7), and (2) we bathed the ganglion in a high divalents solution to abolish them (Fig. 8).

For the first series of studies we stimulated one AE motoneuron with a current step while recording a CV motoneuron and an NS neuron, the membrane potential of which was set at $-20 \mathrm{mV}$ or at $-80 \mathrm{mV}$. Figure $10 \mathrm{~A}$ displays representative recordings performed in normal saline solution, where stimulating the AE 

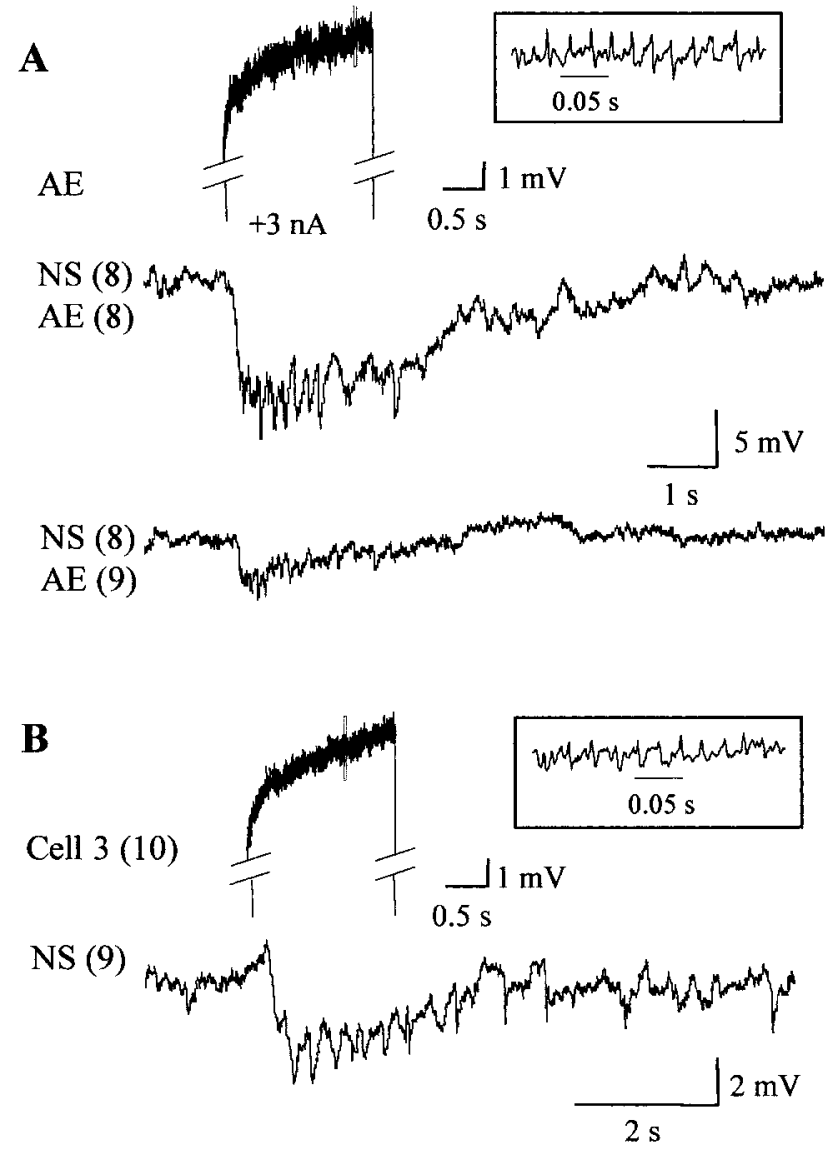

C

Cell $1(10)$
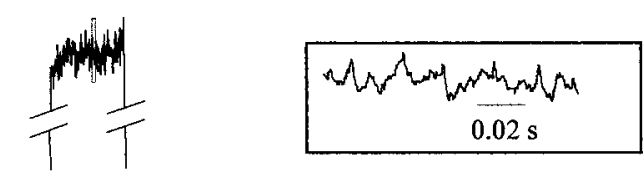

NS (9)

Figure 9. Responses of NS neurons to the excitation of motoneurons in local and adjacent ganglia. Recordings of the responses of NS neurons to the stimulation of different motoneurons, performed in isolated three-ganglia chains (typically from midbody ganglion M7 to M9 or M10 to M12). The notation on the left indicates the identity of the recorded neurons, where the number between brackets designates the ganglion number. $A$, Representative responses of an NS neuron to the stimulation of a local AE motoneuron [NS(8)-AE(8)] and to an AE motoneuron located in the adjacent posterior ganglion $[N S(8)-A E(9)]$. The two $A E$ motoneurons were given identical stimuli ( $+3 \mathrm{nA}$ square current step), and their responses were highly similar. The top trace shows the activity of the posterior $\mathrm{AE}[A E(9)]$ motoneuron during the stimulation period. The inset shows the fragment of the recording indicated by the dotted rectangle in an expanded temporal scale. $B$, Representative recordings showing the response of an NS neuron to the stimulation of a cell 3 in the posterior ganglion. C, Representative recordings showing the response of an NS neuron to the stimulation of a cell 1 in the posterior ganglion.

motoneuron increased the firing frequency of the CV motoneuron when the $\mathrm{NS}$ neuron was hyperpolarized to $-80 \mathrm{mV}$ (Fig. $10 \mathrm{Ai}$ ). However, when the NS neuron was held at $-20 \mathrm{mV}$ and displayed a substantial hyperpolarizing synaptic potential, the activation of the AE had no effect on the CV motoneuron (Fig. $10 \mathrm{Aii})$. These results suggest that the $\mathrm{CV}$ and $\mathrm{AE}$ motoneurons were electrically coupled and that this coupling depended on the membrane potential of the NS neuron. The question that remained open was whether the coupling among motoneurons depended directly on the membrane potential of NS neurons or whether it required the activation of the interneurons that transmit the synaptic responses from motoneurons to NS neurons.

To distinguish between these two possibilities, we performed similar experiments in a solution with high divalent cations that impairs the inhibitory potentials of NS neurons. Figure $10 \mathrm{~B}$ displays representative recordings showing that in this condition the coupling between the motoneurons was independent of the membrane potential of the NS neuron.

We quantified the results from these experiments by measuring the coupling between motoneurons and the intensity of the response shown by the NS neurons. The motoneuron coupling was calculated as the change in firing frequency (Fig. 10, legend) of the $\mathrm{CV}$ motoneuron, with respect to its basal firing rate, caused by the stimulation of the AE motoneuron. The magnitude of the response of NS during the $\mathrm{AE}$ stimulation was measured as the time integral (area) during the stimulus period. As shown in Figure 10 Aiii, in normal saline the firing frequency of the $\mathrm{CV}$ neuron increased significantly $(p<0.01)$ when the NS neuron was at $-80 \mathrm{mV}$, but it did not change when the NS neuron was held at $-20 \mathrm{mV}$. As expected, the area of the NS response at these two voltages shifted from a positive to a negative value. In contrast, in the high divalents solution, the firing frequency of the $\mathrm{CV}$ neuron increased significantly $(p<0.001)$ at both NS potentials, although it was significantly larger $(p<0.01)$ when NS was held at $-20 \mathrm{mV}$, and, under these conditions, the area of the response shown by NS remained positive at both potentials (Fig. 10 Biii).

As noted before, the results from Figure 10 suggested that CV and AE motoneurons were electrically coupled. To further confirm this observation, we analyzed the coupling between both neurons by passing depolarizing and hyperpolarizing square current pulses in each cell while recording the other in ganglia maintained in a solution containing a high $\mathrm{Mg}^{2+} / \mathrm{Ca}^{2+}$ ratio, which is known to impair chemical synapses (see Materials and Methods). Figure 11 shows that the motoneurons were linked by an electrical connection that allowed passage of positive and negative signals in both directions.

\section{Discussion}

Both NS neurons function as a unit

The nonrectifying electrical coupling between NS neurons resulted in a high correlation of spontaneous and evoked activity in the two contralateral homologs in a ganglion. When a specific presynaptic neuron was experimentally activated, the synaptic responses of the two NS neurons evidenced a consistent delay that indicated that the NS neuron receiving the ipsilateral input developed the responses with a shorter latency (Figs. $2 B$, 5). The most plausible explanation is that the contralateral NS cell received the synaptic inputs through a double mechanism via branches of the same interneurons that cross over the contralateral side and from the leading NS neuron via the electrical junctions (Szczupak and Kristan, 1995). The combination of chemical and electrical inputs has been considered a mechanism that contributes to synchronization of the activity of neurons (Tamás et al., 2000). Instead, when the spontaneous synaptic activities of the NS cells were compared the cross-correlograms evidenced no delay. This suggests that the spontaneous activity resulted from common simultaneous inputs to both of the neurons.

\section{The coupling between NS neurons and motoneurons was sensitive to the junction potential}

The electrical connection between the NS neurons and the motoneurons showed a voltage sensitivity consistent with the re- 


\section{Normal Saline}

$\mathbf{A i}$

Aii

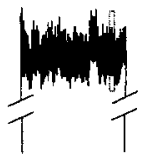

Whathwith

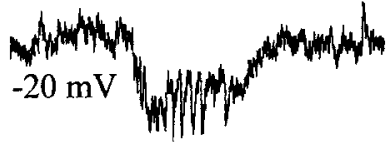

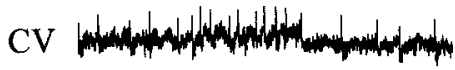

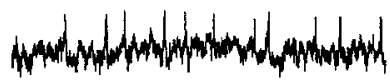

$10 \mathrm{Mg}^{++} / 10 \mathrm{Ca}^{++}$

Bi

Bii

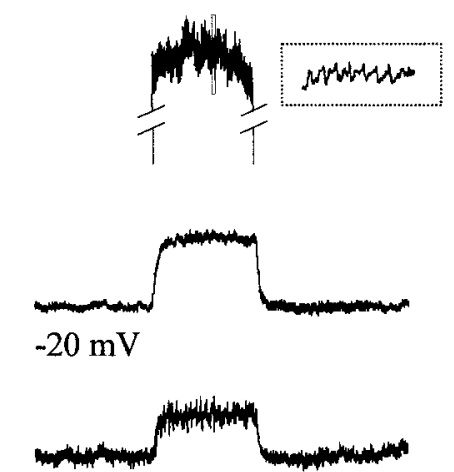

$\mathrm{AE}$

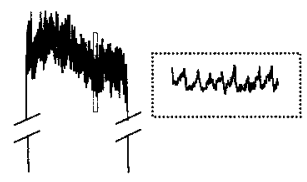

NS

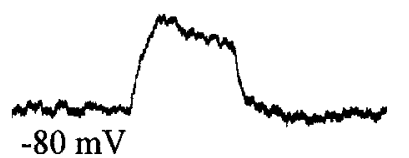

CV
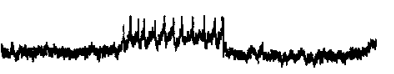

\section{Aiii}
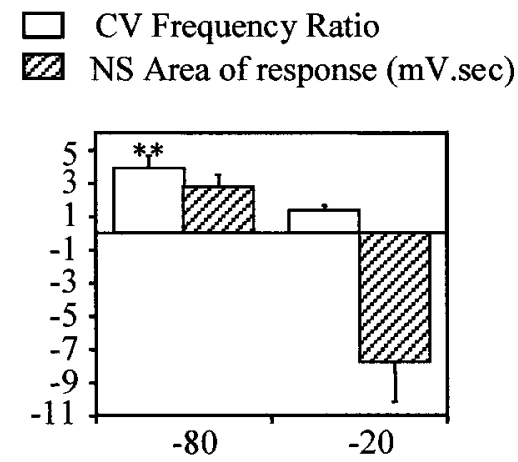

NS Membrane Potential (mV)

Figure 10. NS neurons regulate the coupling between motoneurons. Paired recordings of an NS neuron and a CV motoneuron during the stimulation of a local AE motoneuron with a square current step. Only the part of the recording corresponding to the stimulus is shown for the AE motoneuron. The inset shows the fragment of the recording indicated by the dotted rectangle in an expanded temporal scale. $A$, Representative recordings in normal saline as the membrane potential of the NS neuron was set at $-80 \mathrm{mV}$ (Ai) or at $-20 \mathrm{mV}$ (Aii). The membrane potential of the CV motoneuron was manipulated by injecting DC current to obtain a similar spontaneous firing rate in both conditions. $B$, Experiments performed in a high divalents solution (10 mM $\mathrm{Mg}{ }^{2+} / 10 \mathrm{~mm}$ $\mathrm{Ca}^{2+}$ ) as the membrane potential of the NS neuron was set at $-80 \mathrm{mV}(\mathrm{Bi})$ or $-20 \mathrm{mV}$ (Bii). The graphs show the change in frequency of the CV motoneuron (white columns) and the time integral (area) of the response of the NS neuron (striped columns) for experiments performed in normal (Aiii) and high divalents solution (Biii). The increase in frequency of the CV motoneuron during the injection of current in the AE motoneuron was measured as $f p / f o$, where $f p$ is the firing frequency during the pulse and $f o$ is the basal firing frequency, measured for 13 sec before the stimulation step. The time integral was measured during a period of 4 sec from the beginning of the pulse. The columns and error bars indicate mean and SEM, respectively ( $n=3$ for each column). The magnitude of both parameters is expressed using the same $y$-scale, with the appropriate units specified in column references. ${ }^{*} p<0.01$ and ${ }^{* *} p<0.001$ (compared with the corresponding basal frequency); ${ }^{\S} p<0.01$ (compared with the value at $-80 \mathrm{mV}$ ). Statistical analysis used $t$ tests

ported rectification of the junctions (Wadepuhl, 1989). According to our results, the junction increased its conductance when the motoneuron side was more positive than the NS neuron side. If that were true, then hyperpolarizing the NS neuron should have caused the same effect. Although the results showed a tendency in this direction, the effect of hyperpolarizing the NS neurons was not as marked as depolarizing the motoneuron and was not statistically significant. However, one should notice that the coupling coefficient is not purely a measure of the conductance of the gap connecting the two neurons; instead, it is also a measure of the cell properties (i.e., the path from the soma to the coupling sites, and from there to the soma of the coupled cell). It is possible that manipulating the membrane potential of the NS neurons, at the somatic level, was much more inefficient in influencing the junction region because the transmission depends on passive con- duction. In addition, the observed decrease in the input resistance when the NS cells were hyperpolarized probably exacerbated this condition. When these considerations are taken together, the observed rectification of the electrical NS neuron-motoneuron coupling could be explained in terms of voltage-sensitive gap junctions that would only allow positive current passing from the motoneuron to the NS neuron and only negative current from NS neurons to motoneurons. This feature has a physiologically relevant implication: the effectiveness of the NS neuron-motoneuron interaction was regulated by the activity of the motoneuron, and therefore, the more active the motoneuron, the more susceptible to the hyperpolarizing influence of the NS neuron.

The voltage sensitivity of gap junctions has physiological implications in the function of rectifying synapses (Acklin, 1988; Oh et al., 1999), and it has been postulated as contributing to coincidence 

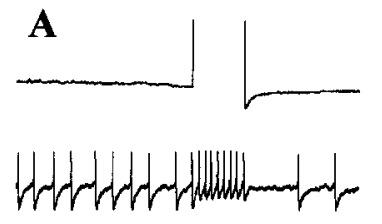

$\mathrm{AE}$

$\mathbf{B}$

CV

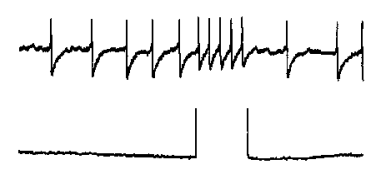

CV

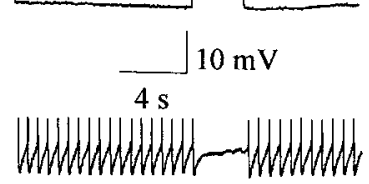

$\mathrm{AE}$
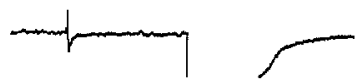

Figure 11. The CV and AE motoneurons are electrically coupled. Paired recordings of an AE and a $\mathrm{CV}$ motoneuron showing the effect of injecting a square current step in one of the neurons, as the ganglion was bathed in a solution with a high $\mathrm{Mg}^{2+} / \mathrm{Ca}^{2+}$ ratio. $A$, The AE motoneuron was stimulated with a $+3 \mathrm{nA}$ pulse (top panel) or a $-3 \mathrm{nA}$ pulse (bottom panel). $B$, The CV motoneuron was stimulated with a $+3 \mathrm{nA}$ pulse (top panel) or a $-3 \mathrm{nA}$ pulse (bottom panel). Qualitatively similar recordings were obtained for another five pairs of $\mathrm{AE}$ and $\mathrm{CV}$ neurons.

detection mechanisms (Edwards et al., 1998). Asymmetries in the transjunctional voltage dependence were observed in heterotypic gap junctions (Bennett et al., 1991), in which the component hemichannels exhibit opposite gating polarities (Verselis et al., 1994). Oh et al. (1999) showed that gap junctions expressed in heterologous systems exhibit a marked dependence of the transjunctional conductance on the junction potential, in the range between -40 and $40 \mathrm{mV}$. This dependence resembles the one observed in our experiments. Interestingly, in the leech the rectification was observed in junctions between nonhomologous cells, whereas homologous neurons showed nonrectifying junctions. It is possible that different leech neurons express innexins with different properties, forming heterotypic gap junctions.

\section{Network and behavioral implications}

The results suggest the existence of a divergent and a convergent network between the NS neurons and the motoneurons. In the divergent path, NS neurons transmit only inhibitory signals to most excitatory motoneurons (Fig. 1), through the rectifying junctions described above. No excitatory signals are transmitted from NS neurons to the motoneurons through these junctions. The convergence of signals transmitted from the motoneurons to the NS neurons takes place along two paths of opposite effects: rectifying electrical synapses transmit depolarizing signals and a polysynaptic path transmits hyperpolarizing signals.

The involvement of interneurons in the latter path is consistent with the fact that stimulation of a motoneuron in one ganglion elicited responses in NS cells of distant ganglia. The motoneuron projections are confined to a single segment, and it is unlikely that NS neurons could transmit a synaptic input passively from their projections in adjacent ganglia (our unpublished data). According to our studies, the interneuronal pathway spanned at least five segments, and thus this network serves as a means to regulate motor activity across ganglia.

Because the magnitude of the inhibitory response depended on the activity of the motoneurons, as the motoneurons became increasingly active the NS neurons showed increasingly larger hyperpolarizing responses. These potentials, in turn, could be transmitted back to the motoneurons through the rectifying junctions and inhibit their activity. This interplay of connections sets the framework for a negative feedback mechanism to curtail

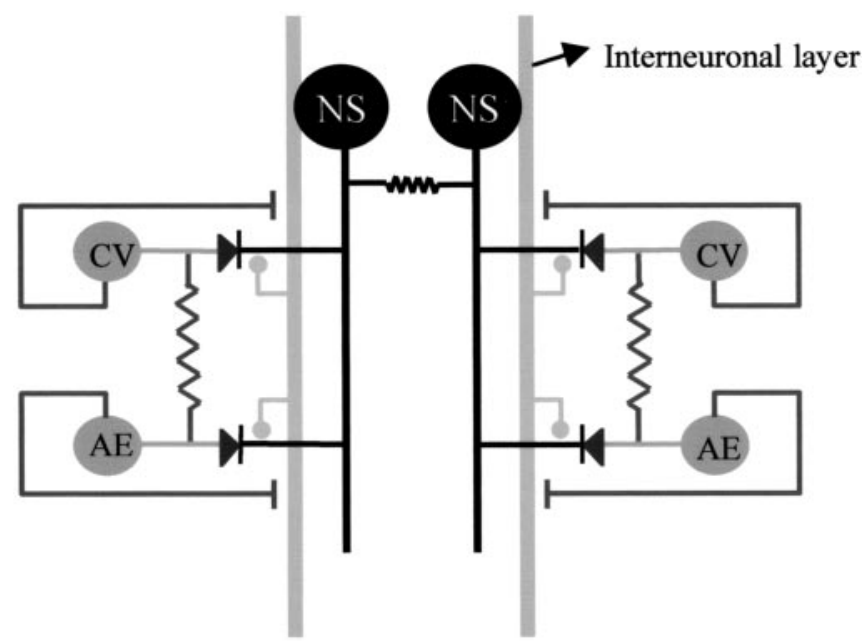

\section{$\longrightarrow$ Inhibitory chemical synapse \\ $\rightarrow$ Excitatory chemical synapse \\ * Rectifying electrical synapse \\ $\rightarrow$ Non-rectifying electrical synapse}

Figure 12. Scheme of the convergent connections between the NS neurons and the motoneurons. The scheme represents a circuit within a single ganglion. The NS neurons (black) are coupled to the motoneurons through rectifying connections, and the AE and CV motoneurons (dark gray) are connected between themselves through nonrectifying junctions. An interneuronal layer (light gray), shown as a single element that spans through the ganglion, along the anteroposterior axis receives excitatory input from the motoneurons and transmits hyperpolarizing signals to the NS neurons. According to this model, these synaptic sites are electrically close to the NS neuron-motoneuron junctions, and thus the hyperpolarizing synaptic potentials could pass to the motoneurons, counteracting the concomitant excitatory effect transmitted through the junctions between the motoneurons.

excessive motor activity, regulated by the membrane potential of the NS neurons. In line with this view, NS neurons neither pass negative current to (Wadepuhl, 1989) nor receive an inhibitory input from inhibitory motoneurons.

This chemically mediated input onto the NS neurons produced a clear-cut influence on the electrical coupling between pairs of nonhomologous motoneurons. It is important to bear in mind that, because of the properties of the rectifying junctions, the NS neuron cannot serve as a bridge to transmit depolarizing signals among the motoneurons connected to it. A putative mechanism to explain the influence of NS neurons on motoneuron coupling is described in Figure 12. In this scheme, the NS neurons are coupled to the motoneurons through a rectifying connection, and the motoneurons are connected among themselves through nonrectifying junctions. An interneuronal layer, represented as a single element that spans through the ganglion, receives excitatory input from the motoneurons and evokes hyperpolarizing signals in the NS neurons. According to this model, these synaptic sites are electrically close to the NS neuron-motoneuron junctions, and thus the hyperpolarizing synaptic potentials could pass to the motoneurons, counteracting the concomitant excitatory effect transmitted through the junctions between the motoneurons.

This model complies with the experimental results in the following way. (1) When the hyperpolarizing synaptic signals were diminished because the NS neuron was set at $-80 \mathrm{mV}$, the signal transmitted passively to the motoneurons was too small to counteract the excitation transmitted from the stimulated motoneuron; and (2) in the absence of the interneuronal activity, no hy- 
perpolarizing signal was initiated in the NS neurons and therefore the coupling took place at any NS potential.

Changing NS membrane potential could have indirectly affected the coupling among motoneurons by modulating the conductance along the rectifying junctions between the NS neurons and the motoneurons: increasing the NS neuron-motoneuron coupling would diminish the effectiveness of the coupling among the motoneurons themselves. However, in normal solution the coupling among motoneurons took place at an NS membrane potential $(-80 \mathrm{mV})$ that enhanced the rectifying junction conductance (Figs. 4, 10). This picture changed in the presence of high divalents, in which the inhibitory synaptic input onto the NS neuron was not expressed. In this condition, the motoneuronal coupling was observed at any NS potential, but it was more effective when the NS neuron was held at a membrane potential at which the conductance of the rectifying junctions was smaller $(-20 \mathrm{mV})$. Taken together the results suggest that the motoneurons were uncoupled by the transmission of the chemically mediated inhibition that the motoneurons themselves induced in the NS neurons.

It is noteworthy that the motoneuronal coupling was not expressed as a one-to-one spike coincidence (Fig. 10). This is in agreement with the observations by Arisi and collaborators (2001) on coactivation of motoneurons during the whole body shortening in the leech (Wittenberg and Kristan, 1992; Shaw and Kristan, 1995), where a significant independence among coactivated motoneurons was found.

Because the NS projections are so widespread and gradual signals are conducted passively, it is highly probable that there is some degree of compartmentalization that defines functional domains in the arborizations. In such a case the NS neuron could function as a distributed regulator of the activity level of semiindependent motor units. Thus, the electrical activity of the NS neuron could reinforce the coactivity of different specific motoneurons, appropriate to the different motor activities that the leech displays. This modulatory action could depend strongly on the spatial distribution of the chemical and electrical sites described in Figure 12 throughout the NS topology. The implementation of imaging techniques will be of crucial importance in studying space distribution of signals throughout the neuritic arbor in different activity patterns.

The effects of the NS neurons on the motor system could be compared, to certain extent, with those of a neuromodulator: they can be exerted on a population of neurons modifying their connectivity properties within a neuronal network. In that sense we consider the NS neuron to be an electrical neuromodulator.

Nonspiking neurons have been also characterized in insects (Burrows, 1992) and share several properties with the leech NS neurons. They are premotor neurons that influence the firing frequency of the motoneurons, they are subjected to predominantly inhibitory signals from premotor spiking interneurons, and they receive sensory input through a layer of spiking interneurons. The most striking difference resides in the fact that the insect nonspiking neurons transmit their signals through chemical synapses.

\section{References}

Acklin SE (1988) Electrical properties and anion permeability of doubly rectifying junctions in the leech central nervous system. J Exp Biol 137:1-11.

Alvarez VA, Chow CC, Van Bockstaele EJ, Williams JT (2002) Frequencydependent synchrony in locus ceruleus: role of electrotonic coupling. Proc Natl Acad Sci USA 99:4032-4036.

Beierlein M, Gibson JR, Connors BW (2000) A network of electrically cou- pled interneurons drives synchronized inhibition in neocortex. Nat Neurosci 3:904-910.

Bennett MV (1997) Gap junctions as electrical synapses. J Neurocytol 26:349-366.

Bennett MV, Barrio LC, Bargiello TA, Spray DC, Hertzberg E, Saez JC (1991) Gap junctions: new tools, new answers, new questions. Neuron 6:305-320.

Burrows M (1992) Local circuits for the control of leg movements in an insect. Trends Neurosci 15:226-232.

De Miguel FF, Vargas-Caballero M, Garcia-Perez E (2001) Spread of synaptic potentials through electrical synapses in Retzius neurones of the leech. J Exp Biol 204:3241-3250.

Dermietzel R (1998) Gap junction wiring: a "new" principle in cell-to-cell communication in the nervous system? Brain Res Brain Res Rev 26:176-183.

Edwards DH, Yeh SR, Krasne FB (1998) Neuronal coincidence detection by voltage-sensitive electrical synapses. Proc Natl Acad Sci USA 95:7145-7150.

Furshpan EJ, Potter DD (1959) Transmission at the giant motor synapses of the crayfish. J Physiol (Lond) 145:289-325.

Galarreta M, Hestrin S (1999) A network of fast-spiking cells in the neocortex connected by electrical synapses. Nature 402:72-75.

Galarreta M, Hestrin S (2001a) Electrical synapses between GABA-releasing interneurons. Nat Rev Neurosci 2:425-433.

Galarreta M, Hestrin S (2001b) Spike transmission and synchrony detection in networks of GABAergic interneurons. Science 292:2295-2299.

Gibson JR, Beierlein M, Connors BW (1999) Two networks of electrically coupled inhibitory neurons in neocortex. Nature 402:75-79.

Granzow B, Friesen WO, Kristan WB (1985) Physiological and morphological analysis of synaptic transmission between leech motor neurons. J Neurosci 5:2035-2050.

Graubard K, Hartline DK (1987) Full-wave rectification from a mixed electrical-chemical synapse. Science 237:535-537.

Hagiwara S, Morita H (1962) Electronic transmission between two nerve cells in leech ganglion. J Neurophysiol 25:721-731.

Iscla I, Arini PD, Szczupak L (1999) Differential channeling of sensory stimuli onto a motor neuron in the leech. J Comp Physiol 184:233-241.

Ishimaru M, Williams JT (1996) Synchronous activity in locus coeruleus results from dendritic interactions in pericoerulear regions. J Neurosci 16:5196-5204.

Kiehn O, Tresch MC (2002) Gap junctions and motor behavior. Trends Neurosci 25:108-115.

Koos T, Tepper JM (1999) Inhibitory control of neostriatal projection neurons by GABAergic interneurons. Nat Neurosci 2:467-472.

Landisman CE, Long MA, Beierlein M, Deans MR, Paul DL, Connors BW (2002) Electrical synapses in the thalamic reticular nucleus. J Neurosci 22:1002-1009.

Mann-Metzer P, Yarom Y (1999) Electrotonic coupling interacts with intrinsic properties to generate synchronized activity in cerebellar networks of inhibitory interneurons. J Neurosci 19:3298-3306.

Marin-Burgin A, Szczupak L (1998) A non-spiking interneuron regulates the sensory input onto the serotonergic neurons in the leech. Soc Neurosci Abstr 645:3.

Marin-Burgin A, Szczupak L (2000) Processing of sensory signals by a nonspiking neuron in the leech. J Comp Physiol [A] 186:989-997.

Muller KJ, Scott SA (1981) Transmission at a direct electrical connexion mediated by an interneurone in the leech. J Physiol (Lond) 311:565-583.

Muller KJ, Nicholls JG, Stent GS (1981) Neurobiology of the leech. Cold Spring Harbor, NY: Cold Spring Harbor Laboratory.

Nicholls JG, Baylor DA (1968) Specific modalities and receptive fields of sensory neurons in CNS of the leech. J Neurophysiol 31:740-756.

Nicholls JG, Purves D (1970) Monosynaptic chemical and electrical connexions between sensory and motor cells in the central nervous system of the leech. J Physiol (Lond) 209:647-667.

Oh S, Rubin JB, Bennett MV, Verselis VK, Bargiello TA (1999) Molecular determinants of electrical rectification of single channel conductance in gap junctions formed by connexins 26 and 32. J Gen Physiol 114:339-364.

Peinado A, Yuste R, Katz LC (1993) Extensive dye coupling between rat neocortical neurons during the period of circuit formation. Neuron 10:103-114.

Schmitz D, Schuchmann S, Fisahn A, Draguhn A, Buhl EH, Petrasch-Parwez 
E, Dermietzel R, Heinemann U, Traub RD (2001) Axo-axonal coupling. a novel mechanism for ultrafast neuronal communication. Neuron 31:831-840.

Shaw BK, Kristan WB (1995) The whole-body shortening reflex of the medicinal leech: motor pattern, sensory basis, and interneuronal pathways. J Comp Physiol [A] 177:667-681.

Stuart AE (1970) Physiological and morphological properties of motoneurones in the central nervous system of the leech. J Physiol (Lond) 209:627-646.

Szczupak L, Kristan WB (1995) Widespread mechanosensory activation of the serotonergic system of the medicinal leech. J Neurophysiol $74: 2614-2623$.
Tamás G, Buhl EH, Lörincz A, Somogyi P (2000) Proximally targeted GABAergic synapses and gap junctions synchronize cortical interneurons. Nat Neurosci 3:366-371.

Verselis VK, Ginter CS, Bargiello TA (1994) Opposite voltage gating polarities of two closely related connexins. Nature 368:348-351.

Wadepuhl M (1987) A morpho- and physiologically uncommon neuron in the leech CNS. Naturwissenschaften 74:43-45.

Wadepuhl M (1989) Depression of excitatory motoneurones by a single neurone in the leech central nervous system. J Exp Biol 143:509-527.

Wittenberg G, Kristan WB (1992) Analysis and modeling of the multisegmental coordination of shortening behavior in the medicinal leech. I. Motor output pattern. J Neurophysiol 68:1683-1707. 